\title{
91. A Theorem on Paracompact Spaces
}

\author{
By Kiyoshi IsÉkI and Yasue MiYanaGa
}

(Comm. by K. Kunugi, M.J.A., June 12, 1956)

Recently, K. Fujiwara [3] and K. Iséki [4] have shown that some properties on compact spaces are generalized very naturally to uniform spaces having Lebesgue property. In this paper, we shall extend a theorem of I. Gelfand and G. Silov [5] to paracompact space.

Let $M$ be a metric space with metric $\rho$, and let $f(x)$ be a function defined on $M$. For a point $x \in M$, we shall define the oscillation of the function $f(x)$ at the point $x$. By $\omega(x, \varepsilon)$, we denote the least upper bound of $\rho(f(p), f(q))$ for $p, q S(x, \varepsilon)$, where $S(x, \varepsilon)$ is the sphere with center at $x$ and radius $\varepsilon$. Then $\lim _{\varepsilon \rightarrow 0} \omega(x, \varepsilon)(=\omega(x))$ exists and this limit is called the oscillation of the function $f(x)$ at the point $x$.

It is well known that a function $f(x)$ defined on a metric space is continuous at a point $x$, if and only if the oscillation of $f(x)$ at $x$ is equal to zero (see W. Sierpinski $[6]$, p. 184).

I. Gelfand and G. Silov [5] proved the following proposition. Let $\varphi(x)$ be a function defined on a compact set $M$ in n-dimensional Euclidean space $R^{n}$, and let $\omega(x) \leq \varepsilon$ for every point $x \in M$, then, there is a continuous function $f(x)$ on $M$ such that $|f(x)-\varphi(x)| \leq 2 \varepsilon$.

We shall extend the proposition by I. Gelfand and G. Silov to more general topological space. First of all, suppose that $M$ is a compact metric space. By the compactness of $M$, we can find a positive number $\eta$ such that $\rho\left(x^{\prime}, x^{\prime \prime}\right)<\eta$ implies $\left|\varphi\left(x^{\prime}\right)-\varphi\left(x^{\prime \prime}\right)\right| \leq 2 \varepsilon$. The open covering $\{S(x, \eta) \mid x \in M\}$ of $M$ has a finite covering $S\left(x_{1}, \eta\right), \cdots$, $S\left(x_{n}, \eta\right)$. Since $M$ is a normal space, for the finite number of the open sets $S\left(x_{i}, \eta\right)(i=1,2, \cdots, n)$, there is such a decomposition $\lambda_{i}(x)$ $(i=1,2, \cdots, n)$ of unity that

(1) each $\lambda_{i}(x)$ is a non-negative, continuous function on $M$,

(2) $1=\sum_{i=1}^{n} \lambda_{i}(x)$ for every $x$ of $M$,

(3) $\lambda_{i}(x)=0$ on $M-S\left(x_{i}, \eta\right)(i=1,2, \cdots, n)$.

(See N. Bourbaki [2], p. 66.) To define a continuous function $f(x)$, let $f\left(x_{i}\right)=\varphi\left(x_{i}\right)$ and

$$
f(x)=\sum_{i=1}^{n} \lambda_{i}(x) f\left(x_{i}\right),
$$

then $f(x)$ is continuous on $M$. For any $x$ of $M$, there is an open sphere $S\left(x_{i}, \eta\right)$ containing $x_{i}$. 


$$
\begin{aligned}
& |\varphi(x)-f(x)|=\left|\varphi(x)-\sum \lambda_{i}(x) f\left(x_{i}\right)\right| \\
= & \left|\sum \lambda_{i}(x)\left(\varphi(x)-f\left(x_{i}\right)\right)\right| \leq \sum \lambda_{i}(x)\left|\varphi(x)-f\left(x_{i}\right)\right| .
\end{aligned}
$$

Since $\rho\left(x, x_{i}\right) \geq \eta$ implies $\lambda_{j}(x)=0$ and $\rho\left(x, x_{i}\right)<\eta$ implies $\left|\varphi(x)-f\left(x_{i}\right)\right|$ $=\left|\varphi(x)-\varphi\left(x_{i}\right)\right| \leq 2 \varepsilon$, we have $|\varphi(x)-f(x)| \leq \sum \lambda_{i}(x)\left|\varphi(x)-f\left(x_{i}\right)\right| \leq 2 \varepsilon$. Therefore we have the following

Theorem 1. Let $\varphi(x)$ be a function defined on a compact metric space $M$ and $\omega(x) \leq \varepsilon$ for every $x$ of $M$, then, we can find a continuous function $f(x)$ such that

$$
|\varphi(x)-f(x)| \leq 2 \varepsilon \text {. }
$$

Now, let $f(x)$ be a function defined on a topological space $M$. By the oscillation of a function $f(x)$ at a point $x$, we shall mean the number $\omega(x)=\inf _{\vee} \delta(f(V))$, where $V$ runs over all neighbourhoods of $x$ and $\delta(f(V))$ is the diameter of $f(V)$.

To extend Theorem 1, we shall consider a paracompact Hausdorff space $M$. Let $\varphi(x)$ be a function with the oscillation $\omega(x) \leq \alpha$ for every point $x$ of $M$. Let $\varepsilon$ be a given positive number. For each $x$ of $M$, there is a neighbourhood $V(x)$ of $x$ such that $\delta(f(V(x))) \leq \alpha$ $+\varepsilon$. Then $\{V(x)\}_{x \in M}$ is a covering of $M$. By the paracompactness of $M$, the covering $\{U(x)\}_{x \in M}$ has locally finite refinement $\left\{U_{\alpha}\right\}$. As well known, for the covering $\left\{U_{\alpha}\right\}$, there is a decomposition of unity:

(4) There are non-negative continuous functions $f_{\alpha}(x)$ for each $\alpha$.

(5) $f_{\alpha}(x)=0$ on $M-U_{\alpha}$.

(6) $\sum_{\alpha} f_{\alpha}(x)=1$ for every $x$ of $M$.

(See, cf. R. G. Bartle and L. M. Graves [1], p. 401.)

Let $x_{\alpha}$ be a point of $U_{\alpha}$, and let

$$
f^{\prime}(x)=\sum_{\alpha} f_{\alpha}(x) \varphi\left(x_{\alpha}\right)
$$

then $f(x)$ is continuous on $M$. For a point $x$ of $M$, we have

$$
\begin{aligned}
& |\varphi(x)-f(x)|=\left|\varphi(x)-\sum_{\alpha} f_{\alpha}(x) \varphi\left(x_{\alpha}\right)\right| \\
= & \left|\sum f_{\alpha}(x)\left(\varphi(x)-\varphi\left(x_{\alpha}\right)\right)\right| \leq \sum_{\alpha} f(x)\left|\varphi(x)-\varphi\left(x_{\alpha}\right)\right| .
\end{aligned}
$$

Suppose that $x \in U_{\alpha_{i}}(i=1,2, \cdots, k)$, then

$$
\left|\varphi(x)-\varphi\left(x_{\alpha_{i}}\right)\right| \leq \alpha+\varepsilon
$$

and, by (5), $f_{\beta}(y)=0\left(\alpha_{i} \neq \beta\right)$, therefore

Hence we have the following

$$
|\varphi(x)-f(x)| \leq \alpha+\varepsilon \text {. }
$$

Theorem 2. Let $M$ be a paracompact Hausdorff space, and $\varphi(x) a$ function on $M$ with $\omega(x) \leq \alpha$. For a given positive number $\varepsilon$, there $i s$ a continuous function $f(x)$ such that

$$
|f(x)-\varphi(x)| \leq \alpha+\varepsilon \text {. }
$$

Some of the results of similar type for vector space valued functions have been obtained by S. Kasahara. The detail considera- 
tions will be appeared in his later paper.

\section{References}

[1] R. G. Bartle and L. M. Graves: Mappings between function spaces, Trans. Amer. Math. Soc., 72, 400-413 (1952).

[2] N. Bourbaki: Topologie Générale, Chap. IX, Hermann, Paris.

[3] K. Fujiwara: Une note sur l'espace produit d'un espace topologique par luimême, Math. Jour. Okayama Univ., 5, 121-125 (1956).

[4] K. Iséki: Notes on topological spaces. II. Some properties of topological spaces with Lebesgue property, Proc. Japan Acad., 32, 171-173 (1956).

[5] I. Gelfand and G. Silov: Über verschiedene Methoden der Einführung der Topologie in die Menge der maximalen Ideale eines normierten Ringes, Recueil Math., 51, 59-73 (1941).

[6] W. Sierpiński: General Topology (2nd Ed.), Toronto (1952). 